\title{
SATELLITE-MAGNETOSPHERE INTERACTIONS
}

\author{
W.-H. IP \\ Max-Planck-Institut für Aeronomie \\ D-37191 Katlenburg-Lindau, Germany
}

\begin{abstract}
One important aspect of satellite-magnetosphere interactions concerns surface modification effects resulting from ion sputtering and/or electrostatic transport. For example, the Galileo measurements showed that the net mass loss rate at Europa could be as much as 40 meters of material per 100 million years. Magnetospheric ion-sputtering effects therefore have the interesting potential of providing an independent method to date the age of Europa's surface. Ion sputtering can also lead to the ejection of large amounts of $\mathrm{H}$ atoms and $\mathrm{H}_{2}$ molecules into the Jovian magnetosphere as well as the formation of extended oxygen exospheres in the vicinity of the Galilean icy satellites. The newly discovered ionosphere of Europa could be of sputtering origin as well.
\end{abstract}

\section{Introduction}

The Galilean satellites are the major source of the magnetospheric plasma in the Jovian system. The Voyager mission showed conclusively that the chemical composition and dynamics of the Jovian magnetosphere are fundamentally determined by the gaseous material emitted from Io (Belcher 1983; Krimigis and Roelof 1983). What had not been realized before the Galileo mission was that other Galilean moons, namely, Europa, Ganymede, and Callisto, all have sizable exospheres and hence are potentially important contributors to the Jovian magnetospheric plasma (Barth et al. 1997). The injection mechanisms include Jeans escape of the atomic and molecular hydrogen, ionospheric pickup, and surface ion sputtering. The last two processes depend closely on the plasma parameters in the vicinity of the satellites (i.e., plasma flow geometry, charged particle fluxes, and magnetic field configuration). The Galileo measurements have shown that there are basically three different types of satellite-magnetosphere interactions represented by (a) Callisto, which has no intrinsic magnetic field, (b) Io and Europa, for which magnetohydrodynamic interaction can produce strong field-aligned current systems connecting the satellites to the Jovian ionosphere (Ness et al. 1979; Neubauer 1980), and (c) Ganymede, which has a significant intrinsic magnetic field (Kivelson et al. 1996; Gurnett et al. 1996). We will describe briefly in the following these three prototypes of satellite-magnetosphere interactions and the implications on the surface evolution of the Galilean satellites.

\section{Callisto}

Because of the lack of a shielding ionosphere, the surface of Callisto may be directly subject to the electrostatic charging effect of the magnetospheric plasma. That is, dust grains of sub-micron size could be ejected into ballistic orbits when charged to a surface potential of a few volts (Ip 1986). Such a transport mechanism could serve to disperse surface material over areas not favorable to gravity-assisted motion. However, such an electrostatic levitation effect is critically dependent on the local topographical variations down to a scale of a few meters (if not smaller). It is difficult to assess its cumulative effect over the geological history of Callisto. In any event, if both ion sputtering and thermal sublimation are not important in surface modification, the above-mentioned electrostatic levitation process could be of long-term consequence. 


\section{Ganymede}

The polar regions of Ganymede are subject to the influx of the magnetospheric energetic electrons and ions. Ion sputtering will be the dominant mass erosion effect in these areas where the thermal sublimation rate is very low. From the Energetic Particle Detector (EPD) experiment, the total gas production rates from surface sputtering by ions with energies $>20 \mathrm{keV}$ are $Q\left(\mathrm{H}_{2} \mathrm{O}\right) \sim 2 \times$ $10^{26}$ molecules/s, $Q\left(\mathrm{O}_{2}\right) \sim 1.2 \times 10^{26}$ molecules/s, $Q\left(\mathrm{H}_{2}\right) \sim 1.3 \times 10^{26}$ molecules $/ \mathrm{s}$, and $Q(\mathrm{H}) \sim$ 2.2. $\times 10^{26}$ atoms $/ \mathrm{s}$ (Ip et al. 1997a). Because of the excess kinetic energy at sputtering, the $\mathrm{H}$ atoms and $\mathrm{H}_{2}$ molecules will escape from Ganymede, while most of the $\mathrm{H}_{2} \mathrm{O}$ and $\mathrm{O}_{2}$ molecules will be recycled, forming a thin oxygen exosphere (Johnson et al. 1981; Eviatar et al. 1981; Ip 1996). It is important to note that the peak surface temperature near noon was measured to be about 150 $\mathrm{K}$ at the equator (Orton et al. 1996). This will imply a very high sublimation rate with a total gas production rate of nearly $Q\left(\mathrm{H}_{2} \mathrm{O}\right) \sim 10^{29}$ molecules/s if Ganymede's surface is half-covered by pure water ice. It is still to be investigated how the global sublimation process might be affected by the variations of the surface albedo and chemical composition.

A combination of magnetospheric ion-sputtering effects near the poles and sublimation-driven erosion of the surface material in the low-latitude region will act to shape the surface topography of Ganymede. For example, with a water sublimation rate as high as $5 \times 10^{13} \mathrm{H}_{2} \mathrm{O}$ molecules/s near the equator, the corresponding erosion rate will be as much as $3 \mathrm{~km}$ per 100 million years! This shows, on the one hand, the importance of the sublimation-driven erosion process on Ganymede, and on the other hand, the necessary restructuring of the surface material to be more resistant to thermal sublimation.

The polar cap regions of Ganymede are, in principle, less affected by mass erosion. However, the formation of a field-aligned electric field able to accelerate ionospheric ions to keV energy in the downward direction could lead to an enhanced sputtering effect (Ip et al. 1997b). Furthermore, the sputtered hydrogen atoms and molecules can escape directly, thus producing an asymmetric outflow of hydrogen gas. Such feature might be observable by the ultraviolet spectrometer on Galileo.

\section{Europa}

Because the peak surface temperature is only $120 \mathrm{~K}$ at Europa, thermal sublimation is relatively unimportant in comparison with ion sputtering. A preliminary analysis of the EPD data indicated that the maximum ion sputtering rate from surface impact of energetic ions and the corotating thermal ions are: $Q\left(\mathrm{H}_{2} \mathrm{O}\right) \sim 7.5 \times 10^{27}$ molecules $/ \mathrm{s}, Q\left(\mathrm{O}_{2}\right) \sim 4.5 \times 10^{27}$ molecules $/ \mathrm{s}, Q\left(\mathrm{H}_{2}\right) \sim$ $4.7 \times 10^{27}$ molecules/s, and $Q(\mathrm{H}) \sim 8.7 \times 10^{27}$ atoms $/ \mathrm{s}$. These numbers suggest that almost 80 $m$ of the surface material on Europa could be eroded away over a time interval of 100 million years. Because almost $50 \%$ of the water and oxygen molecules in the exosphere will be lost via direct ejection, ionospheric pickup, and electron impact dissociation, the net mass loss rate is on the order of $40 \mathrm{~m}$ per 100 million years (Ip et al. 1997b).

The above (albeit maximum) estimates for the time change in the vertical height is comparable to the vertical dimension of a crater with a diameter of $200-400 \mathrm{~m}$. It is therefore hoped that further investigations in the Galileo Europa Mission (GEM) will help to provide more accurate determination of the mass erosion rates at Europa. The ultimate goal is to provide an independent method to evaluate the age of the surface units of this unique moon.

Finally, it should be mentioned that the sputtered oxygen molecules will form an exosphere with a column density of 3 to $4 \times 10^{15}$ molecules $/ \mathrm{cm}^{2}$ and a scale height of about $20 \mathrm{~km}$. The ionization of this oxygen exosphere will create an ionosphere with a plasma wake in the downstream direction. For a total $\mathrm{O}_{2}^{+}$production rate of $2 \times 10^{27}$ ions/s, the average density of the electrons in the ionospheric wake could reach a value of 2 to $4 \times 10^{4} \mathrm{~cm}^{-3}$ at a few hundred $\mathrm{km}$ from the surface. This estimate is in agreement with the recent measurements by the radio occultation experiment on Galileo (Kliore et al. 1997).

\section{References}

Barth, C.A. et al. (1997) Geophys. Res. Lett., in press.

Belcher, J.W. (1983) In: Physics of the Jovian Magnetosphere, A.J. Dessler (Ed.), Cambridge University Press, pp. $68-105$,.

Eviatar, A. et al. (1981) Icarus, 47, pp. 75-83. 
Gurnett, D.A. et al. (1996) Nature, 384, pp. 535-537.

Ip, W.-H. (1986) Geophys. Res. Lett., 13, pp. 1133-1136.

Ip, W.-H. (1996) Icarus, 120, pp. 317-325.

Ip, W.-H. et al. (1997a) Geophys. Res. Lett., in press.

Ip, W.-H. et al. (1997b) Geophys. Res. Lett., in press.

Johnson, R.E. et al. (1981) Science, 212, pp. 1027-1030.

Kivelson, M.G. et al. (1996) Nature, 384, pp. 537-541.

Kliore, A. et al. (1997) Science, 277, pp. 355-358.

Krimigis, S.M. and Roelof, E.C. (1983) In: Physics of the Jovian Magnetosphere, A.J. Dessler (Ed.), Cambridge University Press, pp. 106-156.

Ness, N.F. et al. (1979) Science, 204, pp. 982-987.

Neubauer, F.M. (1980) J. Geophys. Res., 85, pp. 1171-1178.

Orton, G.S. et al. (1996) Science, 274, pp. 389-391. 